\title{
The kinase defective EPHB6 receptor tyrosine kinase activates MAP kinase signaling in lung adenocarcinoma
}

\author{
JUN YU ${ }^{1,2}$, ETMAR BULK $^{1}$, PING JI ${ }^{1}$, ANTJE HASCHER ${ }^{1}$, STEFFEN KOSCHMIEDER ${ }^{1}$, \\ WOLFGANG E. BERDEL ${ }^{1}$ and CARSTEN MÜLLER-TIDOW ${ }^{1}$ \\ ${ }^{1}$ Department of Medicine, Hematology and Oncology, University of Münster, Münster, Germany; \\ ${ }^{2}$ Department of Preclinical Experiment Center, Fourth Military Medical University, Xi'an, P.R. China
}

Received January 28, 2009; Accepted March 13, 2009

DOI: 10.3892/ijo_00000326

\begin{abstract}
Decreased expression levels of EPHB6, a member of the receptor tyrosine kinases (RTKs), are associated with an increased risk of metastasis development in early stage non-small cell lung cancer (NSCLC). However, the signaling properties of the kinase-defective EPHB6 receptor are not well-understood. Here, we show that expression of EPHB6 in A549 lung adenocarinoma cells led to phosphorylation of the MAP kinase ERK. Conversely, siRNA based knockdown of EPHB6 reversed ERK phosphorylation. Intriguingly, EPHB6-induced phosphorylation of ERK was uncoupled by activation of the Elk-1 transcriptional factor. These analyses suggest that kinase defective EPHB6 can lead to MAPK activation.
\end{abstract}

\section{Introduction}

The ERK1/2 (p44/42) mitogen-activated protein kinases (MAPKs) are activated in response to a diverse range of extracellular stimuli including mitogens, growth factors and cytokines (1-3) and are of potential importance in the diagnosis and treatment of cancers (4). MEK1 and MEK2 activate ERK1/p44 and ERK2/p42 by phosphorylating activation loop residues Thr202/Tyr204 and Thr185/Tyr187, respectively $(5,6)$. Several downstream targets of ERK1/2 have been identified, including p90RSK (7) and the transcription factor

Correspondence to: Dr Carsten Müller-Tidow, Department of Medicine A, Hematology and Oncology, University of Münster, Domagkstr. 3, D-48129 Münster, Germany

E-mail:muellerc@uni-muenster.de

Abbreviations: NSCLC, non-small cell lung cancer; MAPK, mitogen-activated protein kinase; ERK, extracellular-signalregulated kinase; DMSO, dimethylsulfoxide; FACS, fluorescenceactivated cell sorting

Key words: EPHB6, receptor tyrosine kinase, non-small cell lung cancer, extracellular-signal-regulated kinase, mitogen-activated protein kinase, Elk-1
Elk-1 $(8,9)$. ERK1/2 are negatively regulated by a family of dual-specificity (Thr/Tyr) MAPK phosphatases, known as DUSPs or MKPs, and pharmacologically by MEK inhibitors such as U0126 and PD98059 (10).

EPH receptors form the largest known subfamily of receptor tyrosine kinases, and to date, the EPH subfamily contains 16 members in vertebrates $(11,12)$. The EPH receptors interact with a family of ligands located on the surfaces of adjacent cells, named Ephrins including Ephrin-As and Ephrin-Bs subgroups. The EPH receptors are also grouped into two classes, EPHA receptors (EPHA1-A10) and EPHB receptors (EPHB1-B6). These groups roughly correspond to the receptor-ligand interaction (i.e., Ephrin-As show binding preference for EPHA receptors, and Ephrin-Bs for EPHB receptors). EPHB6 is a member of the EPH family, expression of which is a favorable prognostic marker in neuroblastoma (13-16), metastatic melanoma $(17,18)$, breast cancer (19), prostate carcinomas (20) and non-small cell lung cancer (NSCLC) (21).

EPHB6 is a kinase-dead receptor tyrosine kinase in that its kinase domain contains several alterations in conserved amino acids and is catalytically inactive. The rather good prognosis conferred by EPHB6 expression in several cancers might therefore depend either on a dominant negative mechanism or on specific signaling properties independent of intrinsic kinase activity. Despite the defect of kinase domain, EPHB6 was reported to be phosphorylated by upstream Src family kinase (22). However, the signaling pathways downstream of EPHB6 are largely unknown. Here, we analyzed the phosphorylation status of ERK MAPK to identify possible signaling roles of EPHB6 in NSCLC.

\section{Materials and methods}

Cell culture. A549 lung adenocarcinoma cells were cultured in Dulbecco's modified Eagle's medium (DMEM, Invitrogen, Carlsbad, CA, USA) supplemented with $10 \%$ fetal calf serum (FCS), $2 \mathrm{mM}$ L-glutamine, $100 \mathrm{U} / \mathrm{ml}$ penicillin and $100 \mu \mathrm{g} / \mathrm{ml}$ streptomycin at $37^{\circ} \mathrm{C}$ in $5 \% \mathrm{CO}_{2}$.

Expression constructs and transfection. The coding region of the human EPHB6 cDNA (base 833-3853 NCBI accession no. NM_004445) was cloned into the pcDNA4 To/myc/hisA expression vector (Invitrogen). Human A549 cells were 


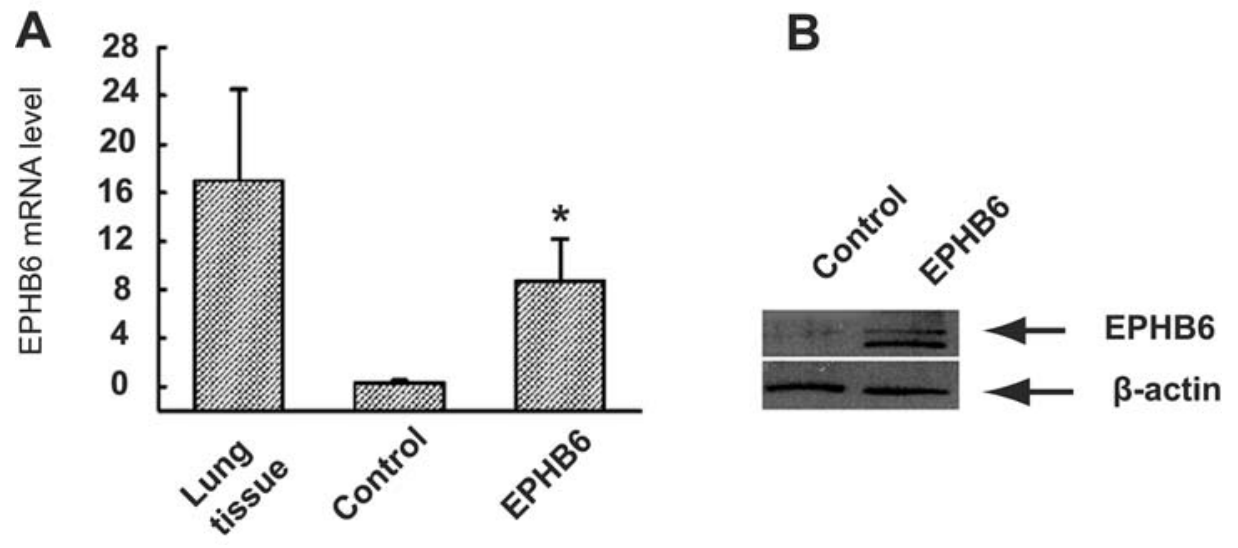

Figure 1. Generation of lung adenocarcinoma cells with EPHB6 re-expression. The expression of the transfected EPHB6 in bulk cultures transfected with either EPHB6 or the empty control vector was shown. (A) Quantitative real-time RT-PCR at mRNA level; and (B) Western blotting at protein level with ß-actin used as loading control. ${ }^{*} \mathrm{P}<0.05$ versus control.

transfected with either EPHB6 expression construct (pcDNA4EPHB6) or empty vector (pcDNA4) using Lipofectamine transfection reagent (Invitrogen). Transfected cells were selected with $400 \mu \mathrm{g} / \mathrm{ml}$ Zeocin (Invitrogen). The expression of EPHB6 was verified by quantitative real-time RT-PCR and Western blotting.

RNA isolation and reverse transcription. Total RNA was isolated using TRIzol reagent (Invitrogen). A total amount of $1 \mu \mathrm{g}$ of RNA from each sample was treated with DNase I, then reverse-transcribed using random primers and MMLV reverse transcriptase according to the protocol of the manufacturer (Promega, Madison, WI, USA).

Western blot analyses. For preparation of whole-cell lysates, cells were washed with ice-cold PBS and lysed for $30 \mathrm{~min}$ on ice in RIPA buffer with $150 \mathrm{mM} \mathrm{NaCl}$ as described (23). Cell lysates were cleared at 20,000 g for $10 \mathrm{~min}$. After adjustment of protein concentrations, the lysates were heated in SDS sample loading buffer at $72^{\circ} \mathrm{C}$ for $10 \mathrm{~min}$ and separated by SDS-polyacrylamide gel electrophoresis (PAGE, 4-12\%, Invitrogen). Gels were blotted on a polyvinylidene difluoride (PVDF) membrane (Immobilon P, Millipore, Bedford, MA, USA) and stained with the indicated first antibody [anti-human EPHB6 (1 $\mu \mathrm{g} / \mathrm{ml}$, From Santa Cruz Inc., Santa Cruz, CA, USA or ABGENT, San Diego, CA, USA), anti-human phosphorylated or total ERK (1:1000 dilution, from Cell Signaling Technology, Inc., Danvers, MA, USA), and antihuman $B$-actin (40 ng/ml, Santa Cruz Inc.). Antibody binding was detected with horseradish peroxidase (HRP)-coupled secondary antibody followed by chemiluminescence detection (ECL Plus; Amersham Pharmacia, Uppsala, Sweden).

PathDetect Trans-Reporter Assay for the activity of Elk-1. The Elk-1-mediated transcriptional activity was assayed using a PathDetect kit (Stratagene, La Jolla, CA, USA). Cells stably expressing pcDNA4 or EPHB6 were seeded in 24-well plates and maintained as described above. The cells were transfected with $1 \mu \mathrm{g}$ of pFR-Luc, $50 \mathrm{ng}$ of pFA2-Elk1 and $0.1 \mu \mathrm{g}$ of pRL-SV40 Vector (Renilla luciferase control vector) as an internal control.
To identify the effects of transient expression of EPHB6, $1 \mu \mathrm{g}$ of pcDNA4-EPHB6 or empty vector pcDNA4 was cotransfected into A549 cells with $1 \mu \mathrm{g}$ of pFR-Luc, $50 \mathrm{ng}$ of pFA2-Elk1 and $0.1 \mu \mathrm{g}$ of pRL-SV40 Vector.

Twenty-four hours after transfection, cells were lysed and the Dual luciferase assay was performed with the Luciferase Reporter Assay Kit (Promega). Data were normalized by the activity of Renilla luciferase reporter vector.

Gene knock-down by siRNA. siRNA for human EPHB6 and scrambled negative control siRNA were both designed, synthesized and labeled with Alexa 555 by Qiagen (Hilden, Germany). Transfection was performed with Lipofectamine 2000 reagent (Invitrogen) and efficiency was tested by fluorescence-activated cell sorting (FACS). The mRNA levels of EPHB6 were detected by quantitative real-time RT-PCR $24 \mathrm{~h}$ after transfection, and protein levels were detected by Western blotting $30 \mathrm{~h}$ after transfection.

Statistical analyses. Quantitative data are presented as mean \pm standard deviation. Statistical analyses were performed using SPSS, version 10.0 (SPSS Science, Chicago, IL, USA). Statistical differences between two independent groups were analyzed by the Student's t-test. P-values with $<0.05$ were considered as significant.

\section{Results and Discussion}

ERK MAPK was potentially activated by EPHB6 in A549 NSCLC cells. To examine the signaling transduction properties of EPHB6 in NSCLC, we established A549 cell lines stably expressing EPHB6 (A549-EPHB6) or the empty vector control (A549-pCDNA4). All experiments were performed with bulk culture cells to preclude clone specific effects. EPHB6 expression was verified by quantitative real-time RT-PCR at the mRNA level (Fig. 1A) and by Western blotting at the protein level (Fig. 1B). The results indicated that EPHB6 expression occurred in a range comparable with normal lung tissue.

In A549-EPHB6 cells, we analyzed STAT5, ERK and AKT by Western blotting for phosphorylation. As a result, STAT5 and AKT were both unaffected (data not shown), whereas ERK 
A

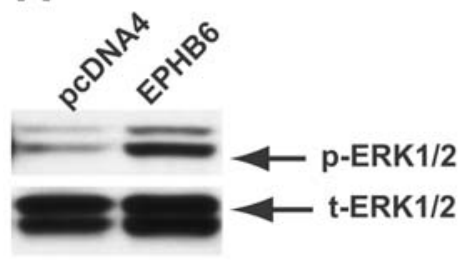

B

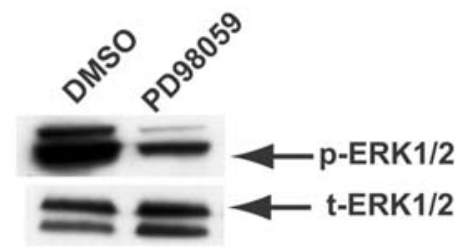

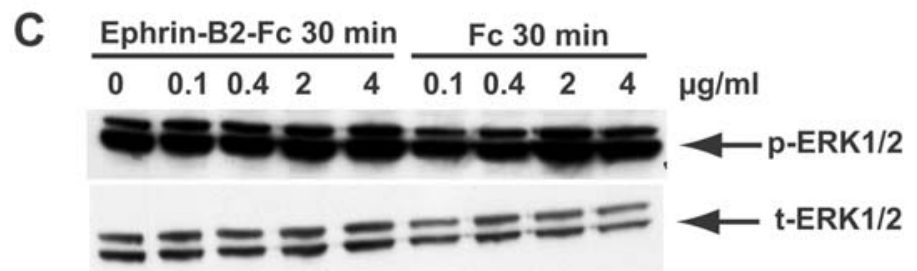

Figure 2. EPHB6 expression leads to ERK MAPK phosphorylation. The phosphorylation of ERK is detected by Western blotting in A549-pcDNA4 or A549-EPHB6 cells. (A) In A549-EPHB6 cells ERK is highly phosphorylated, which could be suppressed by ERK pathway inhibitor $50 \mu$ M PD98059 (B). (C) Ephrin-B2-Fc or naked Fc (R\&D Systems) as control was preclustered by anti-human IgG (Jackson ImmunoResearch Laboratories) at a ratio of 1:2 were applied to the cultured A549-EPHB6 cells. After $30 \mathrm{~min}$, in the concentration from 0 to $4 \mu \mathrm{g} / \mathrm{ml}$, neither Ephrin-B2-Fc nor Fc altered the constitutive activation of ERK.

was strongly activated (Fig. 2A), which could be suppressed by PD98059 (Fig. 2B), a specific ERK pathway inhibitor. Furthermore, ERK phosphorylation was induced in a ligandindependent manner. EPHB6 expression in the absence of Ephrin-B2 ligand still conferred constitutive activation of ERK (Fig. 2C).

To further confirm the association between EPHB6 and ERK, we performed additional siRNA experiments. Since all established lung cancer cell lines expressed low levels of EPHB6 (data not shown), we used siRNA to suppress EPHB6 in A549-EPHB6 cells. EPHB6-siRNA repressed EPHB6 mRNA levels by $\sim 40 \%$ (Fig. 3A), and protein expression was repressed as well (Fig. 3B). The siRNA mediated suppression of EPHB6 resulted in a marked reduction of ERK activation indicating that ERK phosphorylation was initiated by the expression of EPHB6 (Fig. 3C).

The aforementioned results suggested that ERK MAPK was a downstream signaling molecule of EPHB6. Our finding that EPHB6 enhances phosphorylation of ERK MAPK is different from a previous report that EphB6 does not activate the MAPK pathway in Jurkat T cells (24). One possible reason for this discrepancy is tissue specificity of EPHB6.

EPHB6-induced phosphorylation of ERK MAPK was uncoupled with the activation of Elk-1. Elk-1 is a direct nuclear target of ERK MAPK. As a transcription factor, Elk-1 is a component of the ternary complex that binds the serum response element and mediates gene activity in response to serum and growth factors $(8,9,25)$. Elk-1 appears to be a direct substrate for activated ERK MAPK and the kinetics of Elk-1 phosphorylation and activation correlate with the activity of ERK. Also, interfering mutants of ERK blocked Elk-1 activation in vivo $(26,27)$.

To determine whether EPHB6 affects the activation of Elk-1 in A549 cells, we used the PathDetect trans-reporting system, which allows analysis of the pathway with high sensitivity and specificity. In this system, the fusion protein consisting of the activation domain of transcription activator

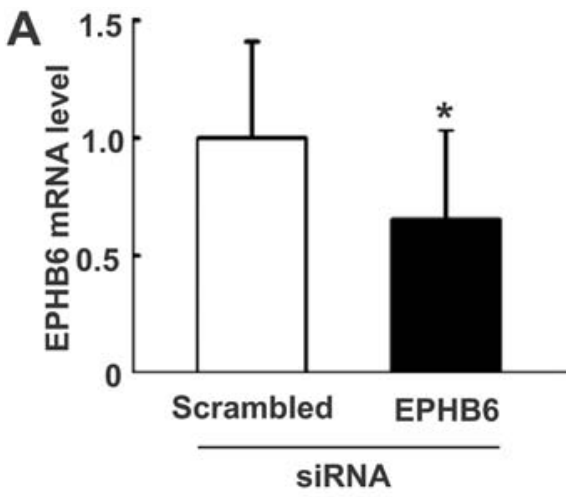

B
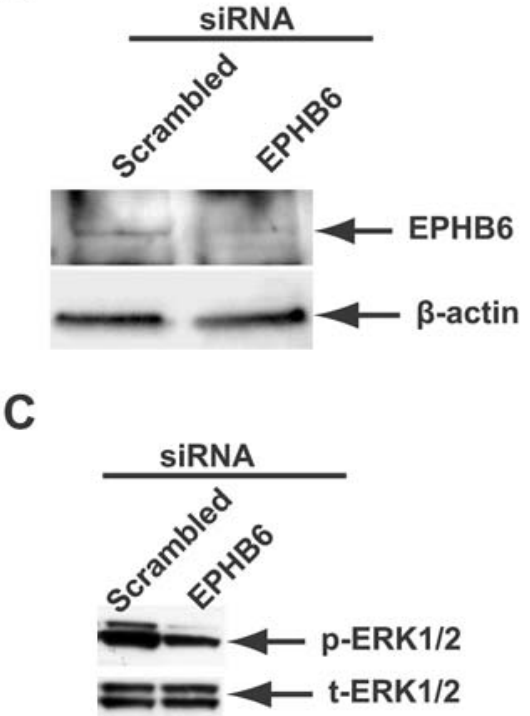

Figure 3. Suppression of EPHB6 by siRNA decreases ERK phosphorylation. (A) A549-EPHB6 cells were transfected with siRNA against EPHB6 or scrambled siRNA as control. EPHB6 mRNA levels were analyzed by quantitative real-time RT-PCR. Data from three independent experiments were analyzed indicating a decrease of $40 \%$ by EPHB 6 specific siRNA ( ${ }^{*} \mathrm{P}<0.05$ versus scrambled control). (B) EPHB6 was also knocked-down by its specific siRNA at protein levels. (C) The phosphorylation status of ERK was determined in EPHB6 siRNA or scrambled siRNA-transfeced cells. 

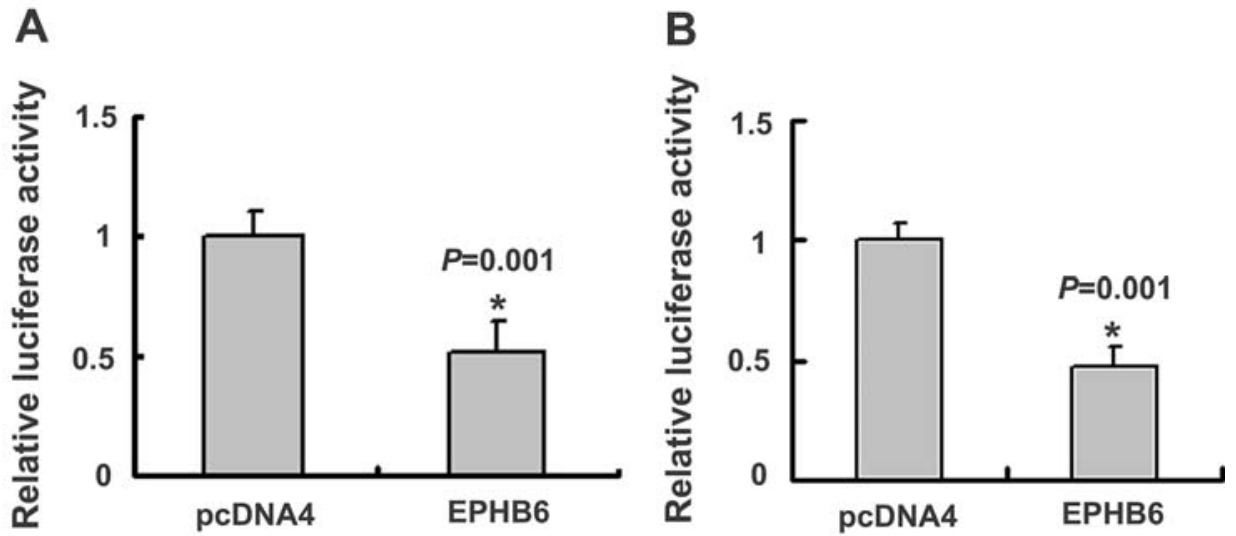

Figure 4. EPHB6 suppresses Elk-1 transcriptional activity. (A) A549-EPHB6 or A549-pcDNA4 cells were transiently transfected with luciferase vectors of pFR-Luc (1 $\mu \mathrm{g})$ and pFA2-Elk1 (50 ng). Renilla luciferase reporter vector (pRL-SV40, 0.1 $\mu \mathrm{g}$ ) was co-transfected as an internal control. (B) A549 NSCLC cells were transiently transfected with an empty vector pcDNA4 $(1 \mu \mathrm{g})$ or an expression vector for EPHB6 (1 $\mu \mathrm{g})$ together with pFR-Luc $(1 \mu \mathrm{g})$ and pFA2-Elk1 (50 ng) along with pRL-SV40 $(0.1 \mu \mathrm{g})$ as an internal control. Twenty-four hours after transfection, cells were lysed and the Dual luciferase assay was performed. Data were normalized by the activity of Renilla luciferase reporter vector, and the arbitrary value of the pcDNA4 transfected cells were set as 1. Data are from triplicate assays in at least 3 independent experiments and are expressed as mean \pm SD. Statistical analyses were based on total observation. ${ }^{*} \mathrm{P}<0.01$ versus empty vector.

and the yeast GAL4 DNA binding domain is analyzed. Luciferase expression is activated when the transcription activation domain of the fusion protein is phosphorylated and the fusion protein binds to the promoter of the luciferase reporter gene containing GAL4 binding sites.

The phosphorylation of ERK due to EPHB6 expression suggested that Elk-1 as a downstream effector would be activated as well. On the contrary, stable expression of EPHB6 significantly attenuated the Elk-1 reporter activity as compared to controls transfected with the empty plasmid (Fig. 4A). Also, the transiently EPHB6-transfected A549 cells elicited a reduction in Elk-1 reporter activity to $\sim 50 \%$ as compared to that obtained with the same dose of the control plasmid (Fig. 4B). Thus, enhanced phosphorylation of ERK by EPHB6 overexpression was not coupled with augmented phosphorylation/activation of Elk-1.

We have recently identified human RTKs whose expression is associated with metastasis development in early stage NSCLC. Expression of several RTKs was associated with an increased frequency of metastasis development. Importantly, EPHB6 was one out of two RTKs associated with a reduced risk of metastasis (21). It is tempting to speculate that the EPHB6 associated uncoupling of signals between ERK and Elk-1 might be related to the favorable role of EPHB6 in NSCLC.

Uncoupling between ERK and Elk-1 activation has been reported, for example, after expression of the kinase suppressor of Ras (KSR) (28), Grb2 associated binder 1 (Gab2) (29) and candidate tumor suppressor Disabled-2 (30). Our finding is also in accordance with a previous report that enhanced NGF-induced phosphorylation of ERK was uncoupled with the activation of Elk-1 in Ndrg4-C2-overexpressed PC12 cell lines (31). The uncoupling of ERK and Elk-1 is acting not unique for EPHB6, but can be induced by several signaling molecules.

Taken together, our current study provides evidence for activation of the ERK MAPK by EPHB6. This activation is not coupled with effective downstream signaling towards Elk-1 transcription factor activity. To the best of our knowledge, EPHB6 is now merely looked upon as a biomarker in certain kinds of tumors (13-21), whereas its functional roles in the tumorigenesis and metastatic process have not been reported systematically. Our findings suggest a role for EPHB6 in cellular signal transduction.

\section{Acknowledgments}

NSCLC research in our laboratory is funded by the Deutsche Forschungsgemeinschaft and the Deutsche Krebshilfe. Part of this work is also supported by the Chinese Natural Science Fund (No. 30801385). We are grateful to Dr Jianping Wu (University of Montreal, Quebec, Canada) for providing EPHB6 cDNA.

\section{References}

1. Roux PP and Blenis J: ERK and p38 MAPK-activated protein kinases: a family of protein kinases with diverse biological functions. Microbiol Mol Biol Rev 68: 320-344, 2004.

2. Baccarini M: Second nature: biological functions of the Raf-1 'kinase'. FEBS Lett 579: 3271-3277, 2005.

3. Meloche S and Pouysségur J: The ERK1/2 mitogen-activated protein kinase pathway as a master regulator of the G1- to S-phase transition. Oncogene 26: 3227-3239, 2007.

4. Roberts PJ and Der CJ: Targeting the Raf-MEK-ERK mitogenactivated protein kinase cascade for the treatment of cancer. Oncogene 26: 3291-3310, 2007.

5. Rubinfeld $\mathrm{H}$ and Seger R: The ERK cascade: a prototype of MAPK signaling. Mol Biotechnol 31: 151-174, 2005.

6. Murphy LO and Blenis J: MAPK signal specificity: the right place at the right time. Trends Biochem Sci 31: 268-275, 2006.

7. Dalby KN, Morrice N, Caudwell FB, Avruch J and Cohen P: Identification of regulatory phosphorylation sites in mitogenactivated protein kinase (MAPK)-activated protein kinase-1a/ p90rsk that are inducible by MAPK. J Biol Chem 273: 1496-1505, 1998.

8. Marais R, Wynne J and Treisman R: The SRF accessory protein Elk-1 contains a growth factor-regulated transcriptional activation domain. Cell 73: 381-393, 1993.

9. Kortenjann M, Thomae O and Shaw PE: Inhibition of v-rafdependent $\mathrm{c}$-fos expression and transformation by a kinasedefective mutant of the mitogen-activated protein kinase Erk2. Mol Cell Biol 14: 4815-4824, 1994. 
10. Owens DM and Keyse SM: Differential regulation of MAP kinase signalling by dual-specificity protein phosphatases. Oncogene 26: 3203-3213, 2007.

11. Menzel P, Valencia F, Godement P, Dodelet VC and Pasquale EB: Ephrin-A6, a new ligand for EphA receptors in the developing visual system. Dev Biol 230: 74-88, 2001.

12. Eph Nomenclature Committee: Unified nomenclature for Eph family receptors and their ligands, the ephrins. Cell 90: 403-404, 1997.

13. Tang XX, Evans AE, Zhao H, Cnaan A, Brodeur GM and Ikegaki N: Association among EPHB2, TrkA, and MYCN expression in low-stage neuroblastomas. Med Pediatr Oncol 36: $80-82,2001$

14. Tang XX, Evans AE, Zhao H, et al: High-level expression of EPHB6, EFNB2, and EFNB3 is associated with low tumor stage and high TrkA expression in human neuroblastomas. Clin Cancer Res 5: 1491-1496, 1999.

15. Tang XX, Zhao $\mathrm{H}$ and Robinson ME: Prognostic significance of EPHB6, EFNB2, and EFNB3 expressions in neuroblastoma. Med Pediatr Oncol 35: 656-658, 2000.

16. Tang XX, Zhao H, Robinson ME, et al: Implications of EPHB6, EFNB2, and EFNB3 expressions in human neuro-blastoma. Proc Natl Acad Sci USA 97: 10936-10941, 2000.

17. Hafner C, Bataille F, Meyer S, Becker B, Roesch A, Landthaler M and Vogt T: Loss of EphB6 expression in metastatic melanoma. Int J Oncol 23: 1553-1559, 2003.

18. Hafner C, Schmitz G, Meyer S, et al: Differential gene expression of Eph receptors and ephrins in benign human tissues and cancers. Clin Chem 50: 490-499, 2004.

19. Fox BP and Kandpal RP: Invasiveness of breast carcinoma cells and transcript profile: Eph receptors and ephrin ligands as molecular markers of potential diagnostic and prognostic application. Biochem Biophys Res Commun 318: 882-892, 2004.

20. Fox BP, Tabone CJ and Kandpal RP: Potential clinical relevance of Eph receptors and ephrin ligands expressed in prostate carcinoma cell lines. Biochem Biophys Res Commun 342: 1263-1272, 2006.
21. Muller-Tidow C, Diederichs S, Bulk E, et al: Identification of metastasis-associated receptor tyrosine kinases in non-small cell lung cancer. Cancer Res 65: 1778-1782, 2005.

22. Matsuoka H, Obama H, Kelly ML, Matsui T and Nakamoto M: Biphasic functions of the kinase-defective Ephb6 receptor in cell adhesion and migration. J Biol Chem 280: 29355-29363, 2005.

23. Tickenbrock L, Schwable J, Wiedehage M, et al: Flt3 tandem duplication mutations cooperate with Wnt signaling in leukemic signal transduction. Blood 105: 3699-3706, 2005.

24. Freywald A, Sharfe N, Rashotte C, Grunberger T and Roifman CM: The EphB6 receptor inhibits JNK activation in T lymphocytes and modulates $\mathrm{T}$ cell receptor-mediated responses. J Biol Chem 278: 10150-10156, 2003.

25. Hill CS and Treisman R: Transcriptional regulation by extracellular signals: mechanisms and specificity. Cell 80: 199-211, 1995.

26. Cavigelli M, Dolfi F, Claret FX and Karin M: Induction of c-fos expression through JNK-mediated TCF/Elk-1 phosphorylation. EMBO J 14: 5957-5964, 1995.

27. Whitmarsh AJ, Shore P, Sharrocks AD and Davis RJ: Integration of MAP kinase signal transduction pathways at the serum response element. Science 269: 403-407, 1995.

28. Sugimoto T, Stewart S, Han M and Guan KL: The kinase suppressor of Ras (KSR) modulates growth factor and Ras signaling by uncoupling Elk-1 phosphorylation from MAP kinase activation. EMBO J 17: 1717-1727, 1998.

29. Zhao C, Yu DH, Shen R and Feng GS: Gab2, a new pleckstrin homology domain-containing adapter protein, acts to uncouple signaling from ERK kinase to Elk-1. J Biol Chem 274: 19649-19654, 1999.

30. Smith ER, Capo-chichi CD, He J, et al: Disabled-2 mediates c-Fos suppression and the cell growth regulatory activity of retinoic acid in embryonic carcinoma cells. J Biol Chem 276: 47303-47310, 2001.

31. Hongo S, Watanabe T, Takahashi K and Miyazaki A: Ndrg4 enhances NGF-induced ERK activation uncoupled with Elk-1 activation. J Cell Biochem 98: 185-193, 2006. 